\title{
Abordagem diagnóstica para acompanhamento de caso de miocardite aguda: um relato de experiência
}

\author{
Diagnostic approach to acute myocarditis case follow-up: an experience report \\ Enfoque diagnóstico del seguimiento de casos de miocarditis aguda: informe de experiencia
}

Barbara Beatriz Lira da Silva

ORCID: https://orcid.org/0000-0001-9166-6147 Universidade Estadual do Piauí, Brasil

E-mail: brbeatriz16@gmail.com

Ana Klara Rodrigues Alves

ORCID: https://orcid.org/0000-0002-1216-9386 Universidade Estadual do Piauí, Brasil

E-mail: klaraphb@outlook.com

Gabriel Brito da Silva

ORCID: https://orcid.org/0000-0002-9765-1074 Faculdade de Ciências Humanas, Exatas e da Saúde do Piauí, Brasil

E-mail: bielgnr@hotmail.com

Flávia Dias Nogueira

ORCID: https://orcid.org/0000-0001-6872-7562

Instituto do Coração, Brasil

E-mail: fla_dn@hotmail.com

Lizandra Azevedo Brito

ORCID: https://orcid.org/0000-0002-5436-3603 Faculdade de Ciências Humanas, Brasil

E-mail: lizazeevedo@gmail.com

José Vieira Amorim Filho

ORCID: https://orcid.org/0000-0002-4929-8253 Faculdade de Ciências Humanas, Brasil

E-mail: vieirajose330@gmail.com

Pedro Henrique Fréres Holanda

ORCID: https://orcid.org/0000-0003-4699-5130 Faculdade de Ciências Humanas, Brasil

E-mail: pedro.phfh1@gmail.com

Ana Carla Souza Menezes

ORCID: https://orcid.org/0000-0002-3829-1646 Universidade Estadual do Piauí, Brasil

E-mail: anacarla66@hotmail.com

Myrcia Ferreira Lopes Nogueira

ORCID: https://orcid.org/0000-0002-1912-464X

Faculdade de Ciências Humanas, Brasil

E-mail: myrcialopes@hotmail.com

Julyana de Souza Araujo

ORCID: https://orcid.org/0000-0003-0109-1146

Faculdade de Ciências Humanas, Brasil

E-mail: julyana.souza.a@gmail.com

Elisa Costa Leitão

ORCID: https://orcid.org/0000-0001-5923-2429

Faculdade de Ciências Humanas, Brasil

E-mail: elisa_leitao@hotmail.com

Vinicius Enrico de Azevedo

ORCID: https://orcid.org/0000-0002-3550-0959

Faculdade de Ciências Humanas, Brasil

E-mail: vinicius_enrico@hotmail.com

Jairo da Costa Souza

ORCID: https://orcid.org/0000-0001-9231-5808

Christus Faculdade do Piauí, Brasil

E-mail: jairocostachrisfapi@hotmail.com

Victor Rocha Santos

ORCID: https://orcid.org/0000-0002-8068-3264

Centro Universitário UNINOVAFAPI, Brasil

E-mail: victoroxas@gmail.com 
Verbena Krieger Rocha Santos

ORCID: https://orcid.org/0000-0002-5901-2255 Universidade Federal do Maranhão, Brasil

E-mail: verbenakrieger@gmail.com

\title{
Resumo
}

Miocardite é uma doença inflamatória cardíaca que pode ocorrer como consequência de infecções (predominantemente por vírus, mas também por outros agentes infecciosos, incluindo bactérias, protozoários e fungos), exposição a substâncias tóxicas (álcool e drogas) e ativação do sistema imunológico, e está incluída entre as cardiomiopatias secundárias na classificação de 1996 da Organização Mundial da Saúde. O presente estudo tem como objetivo relatar a experiência de profissionais da saúde no acompanhamento de um paciente com miocardite aguda, desde do seu primeiro dia de internação até sua alta. Trata-se de um relato de experiência de abordagem crítico-reflexivo de cunho descritivo-compreensivo sobre um caso vivenciado no município de Niteroí - RJ, em um hospital particular, a cerca de um paciente com suspeita inicial de Doença Arterial Coronariana (DAC), sendo posteriormente confirmado o diagnóstico de Miocardite Aguda (MA), em agosto de 2019. A apresentação da miocardite aguda é extremamente variável e frequentemente subclínica, o que pode levar a complicações agudas ou progredir de forma relativamente assintomática para insuficiência cardíaca crônica. O padrão ouro para o diagnóstico de miocardite ainda é a biópsia endomiocárdica positiva, um procedimento invasivo que requer alta probabilidade clínica pré-teste e baixa relação risco / benefício para ser realizado. No entanto, a combinação da medição dos níveis de troponina e ressonância magnética cardíaca tornou o diagnóstico de MA possível com precisão suficiente para formas não complicadas. Ainda existem lacunas na compreensão da patogênese e das opções diagnósticas e terapêuticas para miocardite.

Palavras-chave: Miocardite; Cardiomiopatia; Equipe multidisciplinar.

\begin{abstract}
Myocarditis is an inflammatory heart disease that can occur as a consequence of infections (predominantly by viruses, but also by other infectious agents, including bacteria, protozoa and fungi), exposure to toxic substances (alcohol and drugs) and activation of the immune system. included among secondary cardiomyopathies in the 1996 classification of the World Health Organization. The present study aims to report the experience of health professionals in monitoring a patient with acute myocarditis, from the first day of admission to discharge. This is an experience report of a critical-reflective approach with a descriptive and comprehensive approach on a case experienced in the city of Niteroí - RJ, in a private hospital, about a patient with an initial suspicion of Coronary Artery Disease (CAD), the diagnosis of Acute Myocarditis (AM) was later confirmed in August 2019. The presentation of acute myocarditis is extremely variable and often subclinical, which can lead to acute complications or progress relatively asymptomatically to chronic heart failure. The gold standard for the diagnosis of myocarditis is still positive endomyocardial biopsy, an invasive procedure that requires a high pre-test clinical probability and a low risk/benefit ratio to be performed. However, the combination of measuring troponin levels and cardiac magnetic resonance imaging has made the diagnosis of MA possible with sufficient accuracy for uncomplicated forms. There are still gaps in understanding the pathogenesis and diagnostic and therapeutic options for myocarditis.
\end{abstract}

Keywords: Myocarditis; Cardiomyopathy; Multidisciplinary team.

\section{Resumen}

La miocarditis es una enfermedad cardíaca inflamatoria que puede ocurrir como consecuencia de infecciones (predominantemente por virus, pero también por otros agentes infecciosos, incluidas bacterias, protozoos y hongos), exposición a sustancias tóxicas (alcohol y drogas) y activación del sistema inmunológico. incluida entre las miocardiopatías secundarias en la clasificación de la Organización Mundial de la Salud de 1996. El presente estudio tiene como objetivo reportar la experiencia de los profesionales de la salud en el seguimiento de un paciente con miocarditis aguda, desde el primer día de ingreso hasta el alta. Se trata de un relato de experiencia de abordaje crítico-reflexivo con abordaje descriptivo e integral sobre un caso vivido en la ciudad de Niteroí - RJ, en un hospital privado, sobre un paciente con sospecha inicial de Enfermedad Arterial Coronaria (EAC), el El diagnóstico de miocarditis aguda (MA) se confirmó posteriormente en agosto de 2019. La presentación de la miocarditis aguda es extremadamente variable y, a menudo, subclínica, lo que puede conducir a complicaciones agudas o progresar de forma relativamente asintomática a insuficiencia cardíaca crónica. El estándar de oro para el diagnóstico de miocarditis sigue siendo la biopsia endomiocárdica positiva, un procedimiento invasivo que requiere una alta probabilidad clínica previa a la prueba y una baja relación riesgo / beneficio para realizarse. Sin embargo, la combinación de la medición de los niveles de troponina y la resonancia magnética cardíaca ha hecho posible el diagnóstico de AM con suficiente precisión para las formas no complicadas Todavía existen lagunas en la comprensión de la patogenia y las opciones diagnósticas y terapéuticas de la miocarditis.

Palabras clave: Miocarditis; Cardiomiopatía; Equipo multidisciplinario. 


\section{Introdução}

Miocardite é uma doença inflamatória cardíaca que pode ocorrer como consequência de infecções (predominantemente por vírus, mas também por outros agentes infecciosos, incluindo bactérias, protozoários e fungos), exposição a substâncias tóxicas (álcool e drogas) e ativação do sistema imunológico, e está incluída entre as cardiomiopatias secundárias na classificação de 1996 da Organização Mundial da Saúde. A miocardite tem um amplo espectro de apresentações clínicas e trajetórias, com a maioria dos casos resolvendo-se espontaneamente. É também uma causa relativamente comum de morte súbita cardíaca (MSC) em jovens. Além disso, em alguns pacientes, a inflamação pode causar cicatrizes extensas que desencadeiam a remodelação do ventrículo esquerdo (VE), levando eventualmente a cardiomiopatia dilatada (CMD) ou, alternativamente, a um fenótipo hipocinético não dilatado predominante de cardiomiopatia. A miocardite pode ser caracterizada de acordo com a etiologia, fase e gravidade da doença, sintomas predominantes e achados patológicos (Ammirati et al., 2020).

Novas cardiomiopatias foram descobertas (arritmogênicas, restritivas e não compactadas) e adicionadas para atualizar a classificação da Organização Mundial da Saúde. A miocardite também foi denominada cardiomiopatia inflamatória. O progresso extraordinário realizado na genética molecular das cardiomiopatias hereditárias permitiu o estabelecimento da cardiomiopatia dilatada principalmente como citoesqueleto, doença de transmissão de força; cardiomiopatias hipertróficasrestritivas como sarcômero, doença de geração de força; e cardiomiopatia arritmogênica como desmossomo, doença de junção celular. As canalopatias (QT curto e longo, Brugada e síndromes de taquicardia ventricular polimórfica catecolaminérgica) também devem ser consideradas cardiomiopatias devido à disfunção elétrica dos miócitos (McKenna, Maron e Thiene, 2017).

A miocardite aguda (MA) é uma doença inflamatória do miocárdio que pode ser desencadeada por diferentes estímulos não isquêmicos. Tem um amplo espectro de apresentações clínicas, desde sintomas leves, como mal-estar e dor torácica sem (ou com mínima) disfunção cardíaca, até arritmias com risco de vida e / ou insuficiência cardíaca (IC) grave. Embora os especialistas ainda divirjam entre aqueles que defendem a definição histológica versus aqueles que apoiam uma definição principalmente clínica de miocardite, na prática do mundo real, o diagnóstico de MA sem dúvida mudou de ser baseado principalmente em biópsia para apenas baseado em ressonância magnética cardíaca na maioria dos cenários clínicos. Portanto, é importante definir claramente as configurações selecionadas, pois as informações derivadas da histologia são essenciais para um tratamento ideal (Kang, Ren, Zhao, Ning \& Liu, 2020).

A miocardite apresenta um cenário clínico desafiador devido à sua apresentação heterogênea e ampla apresentações clínicas, desde assintomática até com risco de vida, incluindo morte súbita. Este fato, em conjunto com a falta de métodos diagnósticos não invasivos altamente específicos e sua sobreposição de sintomas com outras doenças cardiovasculares mais prevalentes / incidentes, torna-a uma entidade subdiagnosticada. A suspeita de miocardite pode ser levantada quando os pacientes se queixam de dor torácica ou mesmo de qualquer outro sintoma cardíaco no contexto de mal-estar geral com biomarcadores anormais, alterações no eletrocardiograma (ECG) ou ecocardiograma transtorácico. Isso afeta particularmente aqueles indivíduos que apresentam disfunção ventricular esquerda (VE) de início após infecção viral ou sintomas prodrômicos nas últimas semanas (Wang et al., 2019; Eichhorn et al., 2020).

Frequentemente, a doença arterial coronariana (DAC) deve ser descartada primeiro usando a angiografia coronária. A angiografia coronária não desempenha um papel no diagnóstico de miocardite, exceto para descartar DAC como causa da apresentação clínica, aumento do biomarcador ou anormalidades da modalidade de imagem que podem ser observadas em ambos os processos da doença. Como consequência, um alto nível de suspeita é exigido pelo clínico para decidir descartar miocardite com testes específicos (Palaskas, Lopez-Mattei, Durand, Iliescu \& Deswal, 2020).

A ressonância magnética cardíaca (RMC) foi proposta como uma abordagem confiável com base nos tempos de relaxamento magnético, sugerindo edema miocárdico regional e vazamento de fluido. Os critérios de Lake Louise parecem ser 
um método diagnóstico preciso baseado em evidências de edema miocárdico em imagens ponderadas em T2 mais vazamento capilar em T1(Bracamonte-Baran \& Čiháková, 2017).

O presente estudo tem como objetivo relatar a experiência de profissionais da saúde no acompanhamento de um paciente com miocardite aguda desde o seu primeiro dia de internação até sua alta.

\section{Metodologia}

Trata-se de um relato de experiência de abordagem crítico-reflexivo de cunho descritivo-compreensivo sobre um caso vivenciado no município de Niteroí - RJ, em um hospital particular, a cerca de um paciente com suspeita de Doença Arterial Coronariana (DAC), sendo posteriormente confirmado o diagnóstico de Miocardite Aguda (MA), em agosto de 2019.

É um estudo exploratório-descritivo e avaliativo, onde o instrumento de pesquisa utilizado foi a análise da evolução do paciente com o passar dos dias, somado a realização de entrevista com o mesmo com intuito de verificar seu estado geral e se o mesmo se encontrava satisfeito com atendimento realizado pela equipe. De acordo com Gil (2007), um estudo descritivo tem a finalidade de apresentar as características de uma determinada população ou de um fenômeno, com o objetivo de identificar o perfil do paciente atendido; diagnóstico e causa. Foi realizada também uma pesquisa bibliográfica, fez-se um recorte relacionando ao tema encontrados na base de dados Pubmed, por meio da combinação dos descritores "Myocarditis" and "Diagnosis" and "Risk factors".

\section{Resultados e Discussão}

\section{Descrição do caso}

Paciente deu entrada no hospital no dia 16/08/2019 relatando dor epigástrica há 2 dias, que se intensificou e irradiou para precórdio e dorso. Com histórico familiar de Diabetes mellitus, Hipertensão arterial, Câncer de estômago e doenças cardíacas, porém nega qualquer tipo de comorbidade e alergia. Concomitante, evolui com alterações eletrocardiográfica e marcadores de necrose miocárdica. Em relação aos exames laboratoriais o que chamou bastante a atenção foi: as enzimas cardíacas a creatinofosfoquinase (CPK), troponina e creatina quinase (CK) estavam elevadas sugerindo lesão cardíaca indicando possivelmente um infarto agudo do miocárdio (IAM), proteína C reativa (PCR) que também estava elevado indicando inflamação nas células, sódio e potássio estavam em valores normais. Para confirmar a presença de obstruções das artérias coronárias ou avaliar o funcionamento das valvas e do músculo cardíaco o paciente foi admitido na UTI cardíaca, sendo incialmente submetido coronariografia de urgência, onde foi visto que o mesmo não possuía doença ateromatosa obstrutiva. Equipe seguiu em busca da hipótese diagnóstica (Gräni et al., 2017; Ammirati et al., 2018).

Foi feita uma série de perguntas ao paciente onde o mesmo relatou ter ingerido muita bebida alcoólica uma semana antes e logo em seguida infecção gastrointestinal. Logo, foi realizada uma Ressonância Magnética Cardíaca (RMC) com suspeita de miocardite aguda. Onde foi visto o miocárdio com sinal normal em todas as sequências, observaram-se também focos e áreas com edema esparsos nos segmentos inferior e ínfero-lateral basais, associados a impregnação tardia de contraste epicárdica, de padrão não isquêmico. O aspecto é sugestivo de miocardite aguda (Lurz et al., 2016).

Foi utilizado como tratamento inibidores da enzima conversora de angiotensina, antagonistas da aldosterona e bloqueadores $\beta$-adrenérgicos. Paciente evolui sem recorrência dos sintomas, ou intercorrências no curso da internação, recebendo alta hospitalar dia 22/08/2019 (Ammirati et al., 2018; Tschöpe et al., 2020).

Embora haja um evento adverso especificado "miocardite" na categoria de "distúrbios cardíacos" nos critérios de terminologia comum para eventos adversos, isso não fornece um padrão de como a miocardite é diagnosticada ou tratada. Os artigos que descrevem o uso de estudos de imagem cardíaca ou biópsia endomiocárdica para diagnóstico são limitados a pequenas séries de casos e relatos de casos, que apresentam uma grande variabilidade no uso de qualquer uma das ferramentas 
para o diagnóstico (Mahmood et al., 2018).

A apresentação da miocardite aguda é extremamente variável e frequentemente subclínica, o que pode levar a complicações agudas ou progredir de forma relativamente assintomática para insuficiência cardíaca crônica, iniciando-se clinicamente com complicações crônicas em estágio avançado, como insuficiência cardíaca devido a cardiomiopatia dilatada (CMD). Como consequência, um alto nível de suspeita é exigido pelo clínico para decidir descartar miocardite com testes específicos. O padrão ouro para o diagnóstico de miocardite ainda é a biópsia endomiocárdica positiva, um procedimento invasivo que requer alta probabilidade clínica pré-teste e baixa relação risco / benefício para ser realizado (Cooper et al., 2007; Bracamonte-Baran \& Čiháková, 2017).

Tradicionalmente, a demonstração histopatológica de miocardite tem sido considerada necessária para o diagnóstico de MA, uma vez que a precisão das ferramentas não invasivas era pobre. No entanto, o diagnóstico patológico raramente foi buscado na prática clínica, devido à invasividade, baixa sensibilidade e o valor incremental limitado no que foi considerado na maioria dos casos uma condição autolimitada e benigna. A combinação da medição dos níveis de troponina e RMC tornou o diagnóstico de MA possível com precisão suficiente para formas não complicadas. Ao contrário, em pacientes com MA complicada, e especialmente naqueles que apresentam miocardite fulminante, é necessário um cuidado altamente coordenado para minimizar a morbidade e mortalidade (Ammirati et al., 2021; Tschöpe et al., 2021).

Os níveis de troponina contribuem para a suspeita clínica e diagnóstico. Os valores absolutos da troponina e sua tendência são apenas aproximadamente relacionados com a gravidade e o prognóstico da MA. Embora valores altos ou muito altos devam ser vistos como um marcador de alto risco, o oposto não é verdadeiro com valores moderadamente ou apenas levemente aumentados. Um aumento precoce e um declínio acentuado da troponina geralmente estão associados à resolução ou pelo menos à atenuação do processo inflamatório e a um bom prognóstico (Gilotra et al., 2016; Ganatra \& Neilan, 2018).

O diagnóstico de miocardite aguda permanece um desafio devido à falta de um padrão de referência clínica e à inespecificidade dos sinais e sintomas apresentados e dos achados do ECG. Em centros experientes, a RMC tornou-se uma investigação clínica de rotina; no entanto, a evidência prognóstica atual dessa abordagem é limitada (Chopra et al., 2016; Hung et al., 2016).

\section{Conclusão}

Entrando na década de 2020, ainda existem lacunas em nossa compreensão da patogênese e das opções diagnósticas e terapêuticas para miocardite e cardiomiopatia inflamatória. A disponibilidade de novas técnicas sofisticadas, terapias emergentes e existentes (reaproveitadas), modelagem computacional e novas percepções ajudarão a resolver essas lacunas de conhecimento em um futuro próximo. Abordagens diagnósticas específicas para diferentes cenários clínicos ajudarão a melhorar a subclassificação de pacientes com cardiomiopatia inflamatória e melhorar a terminologia comum em um campo que não é completamente unívoco. Finalmente, a eficácia de muitas terapias existentes, reaproveitadas ou emergentes precisa ser avaliada de forma ampla, controlada.

O diagnóstico desta condição é desafiador devido aos seus sinais e sintomas inespecíficos, falta de padrão de referência de diagnóstico e baixa precisão do eletrocardiograma, ecocardiografia, biomarcadores e até mesmo biópsia endomiocárdica invasiva. Com seus benefícios técnicos de localização de disfunção regional e caracterização de tecido correspondente, a ressonância magnética cardíaca se tornou a principal ferramenta de imagem em muitos centros que caracterizam a gravidade da doença e planejam o manejo do paciente.

A miocardite aguda é uma condição heterogênea com vias fisiopatológicas distintas. Mais pesquisas são obrigatórias para identificar os fatores e mecanismos que podem desencadear / manter ou neutralizar / reparar o dano miocárdico, a fim de fornecer um racional para o futuro tratamento baseado em evidências de pacientes afetados por esta condição. 


\section{Referências}

Ammirati, E., Veronese, G., Bottiroli, M., Wang, D. W., Cipriani, M., Garascia, A., Pedrotti, P., Adler, E. D., \& Frigerio, M. (2021). Update on acute myocarditis. Trends in cardiovascular medicine, 31(6), 370-379.

Ammirati, E., Frigerio, M., Adler, E. D., Basso, C., Birnie, D. H., Brambatti, M., Friedrich, M. G., Klingel, K., Lehtonen, J., Moslehi, J. J., Pedrotti, P., Rimoldi, O. E., Schultheiss, H. P., Tschöpe, C., Cooper, L. T., Jr, \& Camici, P. G. (2020). Management of Acute Myocarditis and Chronic Inflammatory Cardiomyopathy: An Expert Consensus Document. Circulation. 13(11), e007405.

Ammirati, E., Cipriani, M., Moro, C., Raineri, C., Pini, D., Sormani, P., Mantovani, R., Varrenti, M., Pedrotti, P., Conca, C., Mafrici, A., Grosu, A., Briguglia, D., Guglielmetto, S., Perego, G. B., Colombo, S., Caico, S. I., Giannattasio, C., Maestroni, A., Carubelli, V., Metra, M., Lombardi, C., Campodonico, J., Agostoni, P., Peretto, G., Scelsi, L., Turco, A., Di Tano, G., Campana, C., Belloni, A., Morandi, F., Mortara, A., Cirò, A., Senni, M., Gavazzi, A., Frigerio, M, Oliva, F. \& Camici, P. G. (2018). Clinical Presentation and Outcome in a Contemporary Cohort of Patients With Acute Myocarditis: Multicenter Lombardy Registry. Circulation. 11;138(11):1088-1099.

Ammirati, E., Veronese, G., Cipriani, M., Moroni, F., Garascia, A., Brambatti, M., Adler, E. D. \& Frigerio, M. (2018). Acute and Fulminant Myocarditis: a Pragmatic Clinical Approach to Diagnosis and Treatment. Curr Cardiol Rep. 26;20(11):114.

Bracamonte-Baran, W., \& Čiháková, D. (2017). Cardiac Autoimmunity: Myocarditis. Advances in experimental medicine and biology, 1003, $187-221$.

Cooper, L. T., Baughman, K. L., Feldman, A. M., Frustaci, A., Jessup, M., Kuhl, U., Levine, G. N., Narula, J., Starling, R. C., Towbin, J. \& Virmani, R. (2007). American Heart Association; American College of Cardiology; European Society of Cardiology; Heart Failure Society of America; Heart Failure Association of the European Society of Cardiology. J Am Coll Cardiol. 6;50(19):1914-31.

Chopra, H., Arangalage, D., Bouleti, C., Zarka, S.., Fayard, F., Chillon, S., Laissy, J. P., Henry-Feugeas, M. C., Steg, P. G. \& Vahanian, A. (2016). Prognostic value of the infarct- and non-infarct like patterns and cardiovascular magnetic resonance parameters on long-term outcome of patients after acute myocarditis. Int J Cardiol. 1;212:63-9.

Eichhorn, C., Bière, L., Schnell, F., Schmied, C., Wilhelm, M., Kwong, R. Y. \& Gräni, C. (2020). Myocarditis in Athletes Is a Challenge: Diagnosis, Risk Stratification, and Uncertainties. JACC Cardiovasc Imaging. 13(2 Pt 1):494-507.

Ganatra, S. \& Neilan, T. G. (2018). Immune Checkpoint Inhibitor-Associated Myocarditis. Oncologist. 23(8):879-886.

Gräni, C., Eichhorn, C., Bière, L., Murthy, V. L., Agarwal, V., Kaneko, K., Cuddy, S., Aghayev, A., Steigner, M., Blankstein, R., Jerosch-Herold, M., \& Kwong, R. Y. (2017). Prognostic Value of Cardiac Magnetic Resonance Tissue Characterization in Risk Stratifying Patients With Suspected Myocarditis. Journal of the American College of Cardiology, 70(16), 1964-1976.

Gil, A. C. (2007) Como Elaborar Projetos de Pesquis., Atlas, 4(4): 41-57.

Gilotra, N. A., Minkove, N., Bennett, M. K., Tedford, R. J., Steenbergen, C., Judge, D. P., Halushka, M. K. \& Russell, S. D. (2016). Lack of Relationship Between Serum Cardiac Troponin I Level and Giant Cell Myocarditis Diagnosis and Outcomes. J Card Fail. 22(7):583-5.

Hung, Y., Lin, W. H., Lin, C. S., Cheng, S. M., Tsai, T. N., Yang, S. P., \& Lin, W. Y. (2016). The Prognostic Role of QTc Interval in Acute Myocarditis. Acta Cardiologica Sinica, 32(2), 223-230.

Kang, T. D., Ren, Y. L., Zhao, H., Ning, S. Q., \& Liu, W. X. (2020). Risk factors for adverse cardiac events in adults with fulminant myocarditis during hospitalization. World journal of clinical cases, 8(2), 255-263.

Mahmood, S. S., Fradley, M. G., Cohen, J. V., Nohria, A., Reynolds, K. L., Heinzerling, L. M., Sullivan, R. J., Damrongwatanasuk, R., Chen, C. L., Gupta, D., Kirchberger, M. C., Awadalla, M., Hassan, M., Moslehi, J. J., Shah, S. P., Ganatra, S., Thavendiranathan, P., Lawrence, D. P., Groarke, J. D., \& Neilan, T. G. (2018). Myocarditis in Patients Treated With Immune Checkpoint Inhibitors. Journal of the American College of Cardiology, 71(16), $1755-1764$.

McKenna, W. J, Maron, B. J \& Thiene, G. (2017). Classification, Epidemiology, and Global Burden of Cardiomyopathies. Circ Res. 15;121(7):722-730.

Lurz, P., Luecke, C., Eitel, I., Föhrenbach, F., Frank, C., Grothoff, M., Waha, S., Rommel, K. P., Lurz, J. A., Klingel, K., Kandolf, R., Schuler, G., Thiele, H. \& Gutberlet, M. (2016). Comprehensive Cardiac Magnetic Resonance Imaging in Patients With Suspected Myocarditis: The MyoRacer-Trial. J Am Coll Cardiol. 67(15):1800-1811.

Palaskas, N., Lopez-Mattei, J., Durand, J. B., Iliescu, C., \& Deswal, A. (2020). Immune Checkpoint Inibitor Myocarditis: Pathophysiological Characteristics, Diagnosis, and Treatment. Journal of the American Heart Association, 9(2), e013757.

Tschöpe, C., Ammirati, E., Bozkurt, B., Caforio, A., Cooper, L. T., Felix, S. B., Hare, J. M., Heidecker, B., Heymans, S., Hübner, N., Kelle, S., Klingel, K., Maatz, H., Parwani, A. S., Spillmann, F., Starling, R. C., Tsutsui, H., Seferovic, P., \& Van Linthout, S. (2021). Myocarditis and inflammatory cardiomyopathy: current evidence and future directions. Cardiology, 18(3), 169-193.

Wang, Z., Wang, Y., Lin, H., Wang, S., Cai, X., \& Gao, D. (2019). Early characteristics of fulminant myocarditis vs non-fulminant myocarditis: A metaanalysis. Medicine, 98(8), e14697. 